\title{
An Improved Continuation Call-Based Implementation of Tabling
}

\author{
Pablo Chico de Guzmán , Manuel Carro, Manuel V. IIermenegildo , \\ Cláudio Silva, and Ricardo Rocha- \\ School of Conputer Science, Univ. Politénica de Madrid, Spain
}

Depts, of Comp. Science and Electr, and Comp. Eng, Univ, of New Mexico, USA

DCC-FC \& IIACQ; University of Porto, Portugal

\begin{abstract}
Tabled evalualion has been proved an eflective melhod to improve several aspects of goal-oriented query evaluation, including termination and complexity. Several "native" implementations of tabled evaluation have been developol which offer good performance, but many of them require significant changes to the underlying Prolog implementation, including the compiler and the abstract mashine. Approaches based on program transformation, which tend to minimize changes to both the Prolog compiler and the abstract machine, have also been proposed, but they often result in lower efficiency. We explore some techniques aimed at combining the best of these worlds, i.e., developing an extensible implementation which requires minimal modifications to the compiler and the abstract machine, and with reasonably good performance. Our preliminary experiments indicate promising results.
\end{abstract}

Koywords: Tabled logic programming, Implementation, Performance, Program transformation.

\section{Introduction}

Tabling is a resolution strategy which tries to mernoize previous calls and their answers in order to improve several well-known shortcomings found in SLD resolution. It brings some of the advantages of bottom-up evaluation to the top-down, goal-oriented evaluation stratcgy. In particular, cvaluating logic programs under a tabling scheme may achieve termination in cases where SLD resolution does not (bocause of infinite loops - for oxample, the tabled evaluation of bounded term-size programs is guaranteed to always terminate). Also, programs which perform repeated computations can bo greatly sped up. Program declaraliveness is also improved since the order of daluses and goals within a clause is less relevant, if at all. Tabled evaluation has been successfully applicd in many fids, such as deductive databases reasonirige in the seftantio: Web , model checking , program analysis , and ollhers. 
In all cases the advantages of tabled evaluation stem from checking whether calls to tabled predicates, i.e., predicales which have been marked to be evalu ated using tabling, have been made before. Repeated calls to tabled predicates consume answers from a table, they suspend whon all stored answers have boen consumed, and they fail when no more answers can be generaled. However, the advantages are not without drawbacks. The main problem is the complexity of some (efficient) implementations of labled resolution, and a secondary issine is the difficulty in selecting which predicates to table in order not to incur in undesired slow-downs.

Two main categories of Labling mechanisms can be distingulished: suspensionbased and lincar tabling mochanisms. In suspension-based mechanisms the computation state of suspended tabled subgoals has to be preserved to avoid back tracking over them. 'Ihis is done oither by freezing the stacks, as in XSB , by copying to another area, as in CAT , or by using an intermediate solution as in CIIAT . Linear tabling mechanisms maintain a single execution tree where tabled subgoals always extend the curront computation withont roquiring suspersion arde resemption of sub-computations. The computation of the (local) fixpoint is performod by ropoatedly looping subgoals until no more solutions can be fond. Fxamples of this melhod are the linear tabling of B-Proloy and the DRA scheme $[9]$.

Suspension-based mochanism have achioved very good porformance results but, in general, deep changes to the underlying Prolog implementation are required. Lincar mechanisms, on the other hand, can usually be implemented on top of existing sequential engines without major modifications but thein efliciency is affected by subgoal recomputation. One of our theses is that it should be possible to find a combination of the best of both worlds: a susponsion-based mechanism that is reasonably efficient and does not require complex modifications to the compiler or underlying Prolog implementation, thus contributing to its maintainability an making it easier to port it to other Prolog systems. Also, we would like to avoid introducing any overhead that would reduce the execution speed for SLD excoution.

Our starting point is the Continuation Call Mechanism presented by Rarresh and Chen This approach has the advantage that it indeed does not need deep thanges to the underlying Prolog machinery. On the other hand it has shown up to now worse efficiency than the more "native" suspension-based implementations. Our aim is to analyze the bottlenceks of this approach, explore variations thereof, and propose solutions in order to improve its efliciency while keeping tabling-related changes clearly separated from the basic WAM implementation. While the approach may not necessarily be significantly simpler than other (native) approaches, we will argue that it does allow a more modular design which reduces and isolates in separate modules the changes made to the underlying WAM. This hopefully will make it easier to maintain the implementation of both tabling and the WMM itsolf, as well as adapting the tabling schme and code to other Prolog systems. 
In more concrete terms, the implementation wo will propose tries to be non intrusive and change only minimally the initial WAM, moving the low-level tabling data structures either to the Prolog level or to external modules. Other systems, like Mercury also implement tabling using external modules and program transformation, so as not to change the compiler and runtime system. Despite these similarities, the big differences in the base language make the implementation technically very different also.

\section{Tabling Basics}

We now sketch how labled evaluation works frorr a nser point of view and briefly describe the continuation call mechanism implementation technique proposed , on which we basc our work.

\subsection{Tabling by Example}

We use as rumning example the program in Figure 1 , whose purpose is to determine reachability of nodes in a graph We ignore for now tho :- tabled path/2 declaration (which instructs the compiler to use tabled execution for the designated predicale), and assume that SLD resolution is to be esed. Then, a query such as ?- path $(a, N)$. may never terminate if, for example, edge/2 represerts a cyclic graph.

Adding the :- tabled declaration forces the compiler and runtime system to distinguish the first occurrene of a tabled goal (the generator) and subsequent calls which are identical np to variable renaming (the consumers). The generabor applies resolution using the program clauses to derive answers for the goal. Consumers suspend the current execution path (using implementation-dependent means) and start execution on a different branch. When such an alternative branch finally succeds, the answer generated for the initial query is inserted in a table associated with the original goal. This makes it possible to reactivate suspended calls and to continue execution at the point where they were stopped. 'I'hus, consumers do not use SLL) resolution, but obtain instead the answers from the table where they were inserted previously by the producer. Predicates not marked as tabled are executed following SLL) resolution, hopefully with (minimal or no) overhead due to the availability of tabling in the system.

\subsection{The Continuation Call Technique}

The continuation call technique [14] implements tabling by a combination of program transformation and side effects in the form of insertions into and retrievals from a table which relates calls, answers, and the continuation code to be cxccuted after consumers read answers from the table. We will now sketch how the ruechanism works using the path/2 example (Figure 1). The original code is transformed into the program in Figure 2 which is the one actually executed.

Roughly speaking, the transformation for tabling is as follows: a bridge predicale for path/2 is introduced so that aalls to path/2 made from regular Prolog 
:- tabled path $/ 2$.

$$
\begin{aligned}
& \text { path }(X, Z):- \\
& \text { edge }(X, Y), \\
& \operatorname{path}(Y, Z) . \\
& \text { path }(X, Z):- \\
& \text { edge }(X, Z) .
\end{aligned}
$$

Fig. 1. A sample program

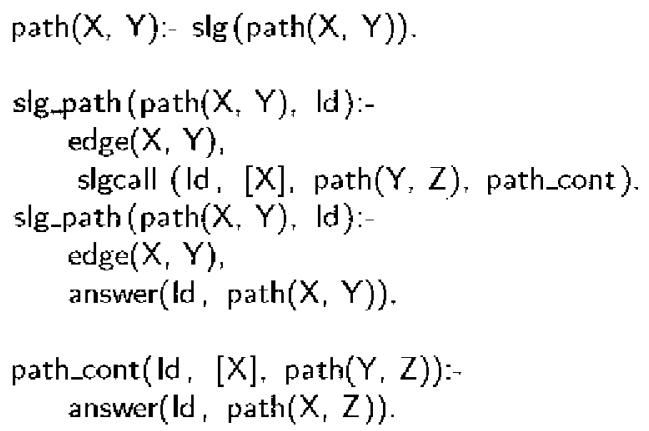

Fig. 2. The program in Figure 1 after being transformed for tabled execution

execution do not need to be aware of the fact lhat path/2 is beirng tabled. The call to the $\mathrm{slg} / \mathrm{I}$ primitive will ensure that its argument is executed to com pletion and will return, on backtracking, all the solutions found for the tabled predicate. To this end, $s \mathrm{~g} / 1$ starls by inserting the call in the arswer table arid generating an identifier for it. Control is then passed to a new, distinct predicate: in this case, slg_path $/ 2 .^{1} \mathrm{slg}$-path/2 recives in the first argument the original call to path/2 and in the second one the identifier generated for the parent call, which is used to relate operations on the table with this initial call. Each clause of slg-path/2 is derived from a clause of the original path/2 predicate by:

- Adding an answer/2 primitive at the end of each clause resulting from a transformation and which is not a bridge to call a continmalion predicate. answer $/ 2$ is responsible for checking for redundant answers and executing whatever continuations (see the following item) thore may be associated with that call identified by its first argument.

- Instrumenting recursive calls to path/2 using the slgcall/4 primitive. If the term passed as an argument (i.e., path $(\mathrm{X}, \mathrm{Y})$ ) is already in the table, slgcall/4 creates a new consumer which consumes answers from the lat ble. Otherwise, the term is inserted in the table with a new call identifier and execution follows using the slg_path/2 program clanses to derive new answers. In the first case, path_cont $/ 3$ is recorded as (one of) the continuation(s) of path (X, Y) and slgcall/4 fails. In the second case path_cont/3 is only recorded as a continuation of path $(X, Y)$ if the tabled call cannot be completed. The path_cont/3 continuation will be called from answer $/ 2$ after inserting a new answer or erased upon completion of path $(\mathrm{X}, \mathrm{Y})$.

- The body of path_cont $/ 3$ encodes what remains of the clause body of path $/ 2$ after the recursive call. It is constructed in a similar way to slg-path/2, i.e., applying the same trantormation as for the initial clauses and calling slgcall/4 and answer $/ 2$ at appropriate times. 


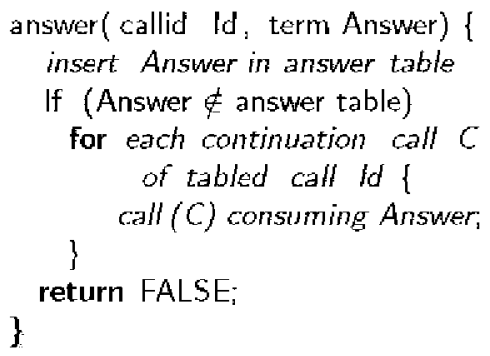

Fig. 3. Pseudo-code for answer $/ 2$

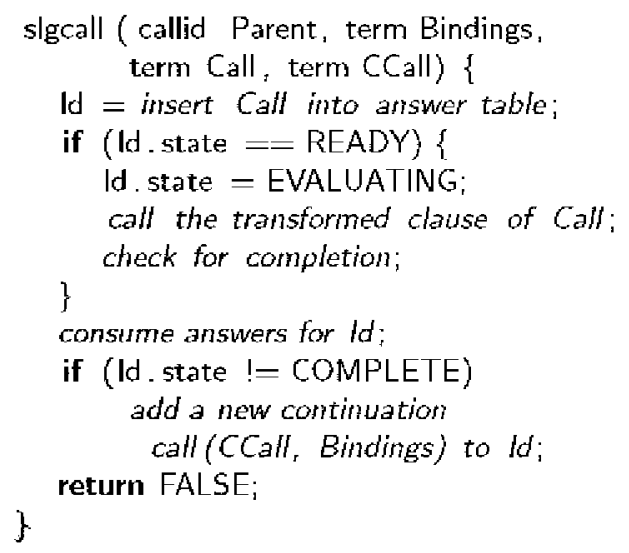

Fig. 4. Pseudo-code for slgcall/4

'The scond argument of slgcall/4 and path_cont/3 is a list of bindings needed to recover the environment of the continualion call. Note that, in the program in Figure 1, an answer to a query such as ?- path (X, Y) may nod to bind variable $X$. This variable does not appear in the rectursive call to path/2, ard hence it does not appear in the path/2 term passed on to slgcall/4 either. In order for the body of path_cont 3 to insert in the table the answor corrosponding to the initial guery, variable $X$ (and, in general, any other necessary variable) bas to be passed down to answer $/ 2$. 'This is done with the list [X], which is inserted in the table as well and completes the enviromment needed for the contination path_cont $/ 3$ to resume the previously suspended call.

$\Lambda$ safo approximation of the variables which should appear in this list is the set of variables which appear in the clause before the tabled goal and which are used in the continuation, including the answer $/ 2$ primitive if there is one in the continuation - this is the case in our example. Variables appearing in the tabled call itself do not need to be included, as they will be passed along anyway.

Recovering a previous execution environment is an important operation in tabled execution. Other approaches to this end are the use of forward trail and freeze registers of SLG-WAM [16], which involves using lower-level mechanisms. The continualion call approach, which performs several habling operations at, the Prolog level through program transfomation and can a priori be expected to be somewhat slower, has, however, the nice property that the implementation does not need to change the underlying WAM machinery, which helps its adaptation it to different $I^{3}$ rolog systems. On the other hand, the table management is usually, and for efficiency reasons, written using some lower-level language and accessed using a suitable interface.

The psendo-code for answer/2 and $s$ gcall/4 is shown in Figures 3 and 4 , respectively. The pseudo code for $\mathrm{sig} / 1$ is similar to that of slgcall/4 but, in stcad of consuming answers, they aro roturnod on backtracking and it finally fails wher all the stored answers have been exhansted. The progra.ru trafsiormation 
and primitives try to complete subgoals as soon as possible, failing whenever new answers are found. Thus, they implement the so-called local scheduling

\section{Checking for completion: The completion detection algorithm}

is similar to that in the SLG-WMM. Wo just provide a sketch liere. Completion is checked for in the execution of the slgcall/4 primitive after exhausting all alternatives for the subgoal call at hand and resuming all of its consumers. To do that, we use two auxiliary fields in the table entry corresponding to every subgoal, SgFr_dfn and SgFr_dep, to quickly determine whether such a subgoal is a leader node. 'The SgFr_dfn field reflects the order in which the subgoals being evaluated were called. New subgoal frames are numbered incrementally as they are created, adding one to the SgFr_dfn of tho previous (youngest) subgoal, whose frame is always pointed to by the global vari able SF_TOP. SgFr_dep holds the number of the older call on which it depends, which is initialized with the same number as SgFr_dfn, rreaning that initially no dependencies exist. If $P_{1}$, a tabled subgoal already inserted in the table, is called from the exceution of another tabled subgoal, $P_{2}$, the SgFr_dep ficld of the table entry of $P_{2}$ is updated with the value of SgFr_dep field of $P_{1}$, meaning $P_{2}$ deponds on $P_{1}$. Whon checking for completion, and using this information from the hable entries, a subgoal can quickly determine whether ib is a leader node. If SgFr_dfn = SgFr_dep, then we know that during its evaluation no dependencios to older subgoals have appoared and thus the Strongly Connected Component (SCC) including the subgoals starting from the table entry referred to by SF_TOP up to the current subgoal can be completed. On the other hand, if SgFr_dep < SgFr_dfn, we annol perform completion. Tnsiedd, we rmet propagate the curent dependency to $C$, the subgoal call that continues the evaluation. 'T'o do that, the SgFr_dep ficld is copicd to SgFr_dep ficld of $C$, and completion can be performed only when the computation readnes the subgoal that does not depend on older stobgals.

Issues in the Continuation Call Mochanism: We have identified two perfor mance-related issues when implementing the technique sketched in the previous section. 'The first one is rather general and related to the hoavy use of the interface between $C$ and Prolog (in both directions) that the implementation makes, which adds an overhead which cannot be neglected.

The second one is the repeated copying of continuation calls. Conlinuation calls (which are, in the end, Prolog predicates with an arbitrarily long list of variables as an argument) are completely copied from P'rolog momory to the table for every consumer fonnd. Storing a pointer to these structures in memory is not onough, sinco slg/1 and slgcall/4 fail immediatcly after associating a continuation call with a tabled call in order to force the program to search for more solutions and complete the tabled call. 'Thorofore, the data structures created during forward execulion may be removed on backl,acking and not be available when needed. Reconstructing continuations as Prolog terms from the data storcd in the table when they are resumed to consume proviously stored answers is recessaly. This car also clearly have a negalive impact on performarice. 
Finally, an issue found with the implementation

is that it did not allow backtracking over Prolog: predicates called from $\mathrm{C}$, which makes it difficult to implement other scheduling strategies. Since this shortcoming may appear also in other $C$ interfaces, it is a clear candidate for improvement.

\section{An Improvement over the Continuation Call Technique}

We now propose some improvements to the different limitations of the original destgrn and implementation that we discussed in Section 2.2. In order to rreasure exceution times, we are taking the implementation doscribed to be close enough to that described in order to be used as a basis for oul developments. It is also an implementation of high quality whose basic components are similar to those in use in current habling systems. This implementation was ported to Ciao, where the rest of the development was performed. In what follows this initial port to Ciao will be termed the "baseline implementation."

\subsection{Using a Lower-Level Interface}

Calls from C to Irolog wre initially performed using a rolatively high-level interface similar to those commonly found in enrent state of the art logic programming systems: operations to create and traverse Prolog terms appear to the programmor as regular $C$ functions, and details of the intornal data representalion are hidden to the programmer. This interface imposed a noticeable overbead in our implementation, as the calls to C functions had to allocate onvironments, pass argurnents, set up Prolog environments to call Prolog from $\mathrm{C}_{2}$ etc.

In order to make our implementation as fast as possible, a possibility is to integrate all the $C$ code into the $W M M$ and try to avoid altogether costly format conversions, etc. However, as mentioned before, we preferred lo make as few changes as possible in the WAM. Therefore we chose to use directly lower-level operations and lake advantage of facilities (e.g., maxros) inilially designed to be internally used by the WAM. While this in principle makes porting more involved, the fact is that the facilitios provided in C interfaces for I'rolog and the internal WAM operations are typically quite related and similar, since they all provide an interface to an underlying architecture and data representation which is common to many Prolog implementations.

Additionally, the code which constructs Prolog terms and performs calls from $\mathrm{C}$ is the same regardless of the program being executed and its complexity is certainly manageable. Therefore, we decided to skip the programmer interface and call directly macros available in the engine implementation. 'That was not a difficult task and it sped the execution up by a factor of 2.5 on average.

\subsection{Calling Prolog from C}

$\Lambda$ relevant issue in the continuation call tochnique (and, possibly, in other cascs) is the use of a C-to-Prolog interface to call Prolog goals from $\mathrm{C}-$ ag-a when 
continuations, which have been internally stored, have to be resumed, as done by $\mathrm{slgcall} / 4$ and answer $/ 2$. We wanted to design a solution which relied as little as possible on non-widely available characteristics of C-to-Prolog interfaces (to simplify porting the code), but which kept the efficiency as high as possible.

The general solution we have adopted is to move calls to contimuations from the $\mathrm{C}$ level to the Prolog level by returning them as a term, using an extra argurnent, in our prinitives, to be called from Prolog. This is possible since continuations are rewritten as separate, unique predicates which therefore have an entry point accossible from Prolog. If several continuations have to be called, they can be returned and invoked one al a lime on backtracking, and fail when there is no pending continuation call. Now continuations generated during program execution can be destructively inserted at the end of the list of continuations transparcntly to I'rolog. Additionally, this avoids using up C stack spaco duc to repealed Prolog $\rightarrow \mathrm{C} \rightarrow$ Prolog $\rightarrow$... calls, which may exhaust the $\mathrm{C}$ stack. Moreover, the $\mathrm{C}$ code is somewhat simplified (e.g., there is no need to set up a I'rolog cnvironmont to bo used from (C) which makes using a lowel-level, faster iriter face less of a burden.

\subsection{Freezing Continuation Calls}

In this section we sketch some proposals to reduce the overhead associated with the way contination calls are haridled in the original continuation call proposal.

Resuming consumers: Our starting point saves a binding list in the lable to reinstall the enviromment of consumers when they have to be resumed. 'I'his is a relatively non-intrusive technique, but it requires copying lerms back and lorth between Prolog and the table where calls are stored. Restarting a consumer needs to construet a term whose first argument is the now answer (which is stored in the heap), the second one is the identifier of the habled goal (an atomic item), and the third one a list of bindings (which may be arbitrarily largo). If the list of bindings has $N$ elements, constructing the contination call requires creating $\approx 2 N+4$ heap cells. If a continuation call is resumed often and $N$ is high, the efficiency of the system can degrade quickly.

The technique we propose construck contimuation calls on the heap as regular Prolog tems. As these continuations are later recovered through a unique call identifier, and etch continuabion is unified with a new, fresh variable (CCall in resume_ccalls/4, Figure 7), full unification or even patterm matching are unnecessary, and resuming a continuation is a constant time operation.

ITowever, the fragment of code which constructs the continuation call performs backtracking to continuc oxploring ponding branches. 'This will remove the constructed call from the heap. Protecting that term is needed to make it possible to constrict it only once and rouse it later. $A$ feasible and simple solution is to freeze continuation calls in a memory area which is not allected by backtracking. 


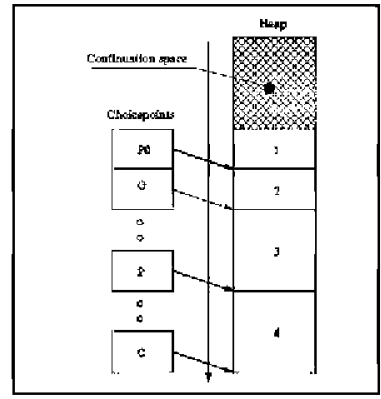

Fig. 5. Luitial state

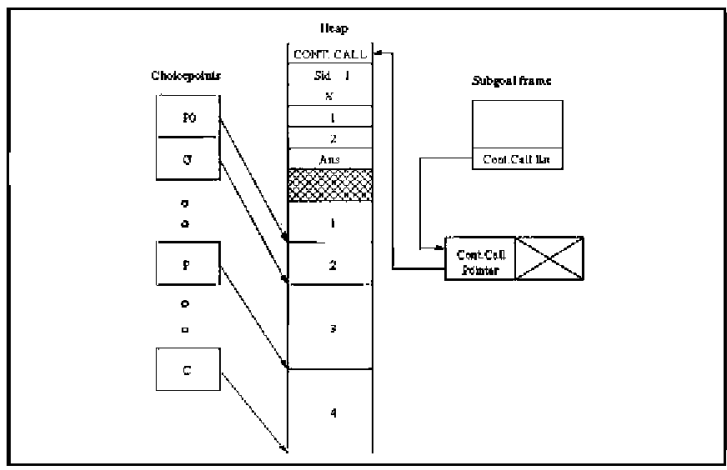

F'g. 6. Frozen continuation call

This will in principle make the aforementioned problem disappear. Selecting a brand new area will, however, bring additional issues as somo WNM instructions would have to be changed in order to lake it into account: for example, variable binding direction is commonly determined using the addrosses of variables (in addition to their tags) so that yourger variables point to older variables in order to save trailing. One easy way to reconcile existing WAM machinery with this continuation call arca is to resorve part of the hoap for it. This makes tho usual WAM assumplions to bold and exactly the same WAM instructions can be nsed to construct and traverse data structures both in the rogular hop and in the continuation call area. Therefore, regarding forward execulion and backtracking, only minimal changes (e.g., the initialization of the $\mathbf{H}$ pointer, and selecting the right read/write heap pointer when doaling with the regular heap or the contimuation call zone) have to be introduced.

Figure 5 shows the state of the choicepoint stack and heap (both assumed to grow downwards) before freezing an contimualion call. Figure 6 stows the continuation call $(C,[X, 1,2]$, Ans) frozen at the beginning of the heap, where it is unaffected by backtracking as the WMM cxcoution startod with the $\mathbf{H}$ pointer placed just after the continuation call zone. In order to recover the continuation calls, a new field is added to the table pointing to a (Prolog) list whose elements, in turn, point to every continuation found so far for a given labied goal.

This makes freezing a continuation call require some extra time in order to copy it on the heap. However, resuming a continuation is a constant time operation. Otter systerns, like CHAT or STC WAM, spend some extra lime while proparing a consumer to be resumed, as they need to record bindings in a forward trail in order to later reinstall them. In our case, when the continuation is to be executed, the list of bindings carried with it is unified with the variables in its body, implementing essentially the same functionality as the forward trail.

In a previous paper [6] we presented a preliminary version of this technique whore the hoap was frozen by manipulating the contents of some choicopoints, 
in what can be seen as a variant of CHAT. The work presented herein works around severat drawbacks in that approach.

Menory management for continuation space: As mentioned before, the area fol continuations is taken from the same memory zone where the general heap is localed, thus making it possible to use the same WAM instructions without any change. In case more memory is needed, reallocating the heap and the contintation area can be done simultaneously, keeping the sarre placement relation between both. As data inside both areas has the same format, adjusting pointers can be dono using momory management routines already oxisting for the regular WAM implementation, which only have to be updated to take into account the cxistence of a gap of unused memory betwoen the continuation call and regular heap areas. Additionally, sliding the heap within its zone to make room for more heap or for more continuations amounts only to readjusting pointers by a cortsharit amourth.

Frozen continuations are, in principle, only reachable from the table struc ture, which makes them candidates to be (wrongly) removed in case of garbage collection. A possible solution which needs almost no change to the garbage oollector is to link a l'rolog list $L$ from some initial, dummy choice point. Each element in $L$ points to the continualion list of a generator, which makes all the continuations reachable by the garbage collector, and therefore protected. When a genorator is completed all of its answors are already stored in tho trie, and its continuations are no longer needed. Removing the pointer from $L$ to this list. of unneeded continuations will make garbage colloction reclaim their space. In order to adjust the pointers from table entries to the continutions when these are reallocated after a garbage collection, each element of $L$ includes a pointer back to the corresponding table cntry which can be used to quickly locate which pointers have to be updated in the lable entries. A new routine has to be added to the garbage collector to perform this step.

Avoiding trail management to recover a continuation call state: The same term $T$ corresponding to a continuation call $C$ can be used several times to generate multiple answers to a query. 'This is in general not a problem as answors are in any case saved in a sale place (e.g., the answer table), and backlracking would undo the bindings to the free variables in $T$. There is, however, a particular case whict reeds special measures. When a continuation call $C_{1}$, identical to $C$, is resumed within the scope of $C$, and it is going to read a new answer, the state of ' $T$ ' has to be reset to its frozen initial state. Since $C_{1}$ is using the same hoap term $T$ as $C$, we say that $C_{1}$ is a reusing call.

'lhe solution wo present trics to oliminate the noed for treating rousing calls as a special case of a continuation call. Reusing calls appear because our baseline implementation resumes continuations when now answers are found, just when we could be in the soope of an identical continuation call. But resumptions can be delayed until the moment in which we are going to check for completion (in the generator) and then the continuation calls with unconsumod answors can be resumed. Following this approach there are no rensing calls becallse a new 
continuation call is never resumed within the scope of another continuation call and we do not need to do any trail management.

New tabling primitives and translation for path/2: Figure 7 shows the new program transfomation we propose for the path/2 program in order to take into account the ideas in the previous sections. Variables Pred, CCall, and F will contain goals built in C but called from Prolog (Section 3.2). The third and fourth arguments of resume_ccalls/4 implement a trick to create a choicepoint with dummy slots which will be used to store pointers to the next continualion to execute and to the generator whose continuations we are resuming. Creating such a slot in this way, at the source level, avoids having to change the structure of choicopoints and how they are mamaged in tho abstract machine.

In the clanse corresponding to path/2, the primilive slg/1 shown in Figure 2 is now split into $s$ gcall/3, execute_generator $/ 2$, and consume_answer $/ 2$. slgcall/3 tosts whether we are in a gencrator position. In that case, it constructs a new goal from the term passed as firsh argument (the term slg-path/2 will bo constructed in this case). 'I'his goal is roturned in variable Pred, which will be called taler. Otherwise, the goal true will be returned.

This new goal is always passed to execute generator $/ 2$ which executes it. If it is true it will succed, and the cxcution will continul with consume_answer $/ 2$. However", slg-path/2 is ensured to nltimalely fail (becaluse the solulions to the tabled predicate are generated by storing answers into the table and failing in answer/2), so that the "else" part of execute_generator/2 is taken. There, consumers are resumed before checking for completion and consume_answer $/ 2$ returns, on backtracking, each of the answers found for path $(X, Y)$.

$\mathrm{slg}$-path $/ 2$ is similar to path/2 but it does not have to return all solutions on backtracking, as consume_answer $/ 2$ does. Instead, it has to generate all possible solutions and save them: new_ccall/5 inserts a new continuation if the execution of $\operatorname{path}(Z, Y)$ is not complete. Otherwise, it uses path_cont_1 as the main functor of a goal whose arguments are answers consumed from the table. This goal is returned in $\mathbf{F}$ and immediately called. In this particular case the (recursive) call to path $/ 2$ is the last goal in the recursive clause (see Figure 1), and therefore the continualion directly inserts the answer in the table.

Finally, answer $/ 2$ does not resume continuations anymore to avoid reusing calls, since resume_ccalls/4 resumes all the continuations of the tabled call identified by Sid and iks dependent generalors before checking for completion.

\subsection{Freezing Auswers}

When resume_ccalls/4 is rosuming continuation calls, answers found for thr tabled calls so far are used to continue execution. These answers are, in prineiple, stored in the table (i.c., answer/2 inserted them), and thoy have to bo constructed on the heap so that the contimulion call carn access them and proceed with execution.

'he ideas in Section 3.3 can be roused to freeze the answers and avot the overthead of building them again. As done with the contimualion calls, a new 
Table 1. 'Terse description of the benchmarks used

\begin{tabular}{|c|c|}
\hline lchain $\mathrm{X}$ & Teft-recursive path program, midimensional graph. \\
\hline Icycle $\mathrm{X}$ & Lefl-recursive palfi program, cyclic graph. \\
\hline rchain $\mathbf{X}$ & $\begin{array}{l}\text { Right-recursive path program (this generates more continuation } \\
\text { calls), unidimensional graph. }\end{array}$ \\
\hline rcycle $\mathbf{X}$ & Right-rocursive path program, cyclic graph. \\
\hline rcycleR $\mathbf{X}$ & Right-ronirsive path program, cyclic graph with repeated odges. \\
\hline reycleF $\mathrm{X}$ & Like rcycle 256 , but executing fib $(20$, ) belore edge $/ 2$ goals. \\
\hline numbors $\mathrm{X}$ & $\begin{array}{l}\text { Find arithmetic expressions which evaluate to some number } N \\
\text { using all the numbers in a list } L \text {. }\end{array}$ \\
\hline numbers $\mathrm{Xr}$ & $\begin{array}{l}\text { Same as above, but all the numbers in } L \text { are all the same (this } \\
\text { generates a larger soarch space). }\end{array}$ \\
\hline atr2 & A parser for . .apancse. \\
\hline
\end{tabular}

Table 2. Speed comparison of three Ciao implementations

\begin{tabular}{|l|r|r|r|}
\hline Benchmark & Ciao + Ccal (baseline) & Lower C interf. Ciao + CC \\
\hline \hline chain 1,024 & 7.12 & 2.85 & 1.80 \\
\hline leycle 1,024 & 7.32 & 2.92 & 1.96 \\
\hline rchain 1,024 & $2,420.60$ & $1,046.10$ & 557.92 \\
\hline rcycle 1,024 & $8,6183.10$ & $2,772.60$ & $1,097.26$ \\
\hline numbers 5 & $1,691.00$ & 781.40 & 772.10 \\
\hline numbers 5r & $3,974.90$ & $1,425.48$ & $1,059.93$ \\
\hline
\end{tabular}

and treasured thee varantis: the fist one is based on a direct adaplation of the implementation presented , using the standard, high-level C interface. Wo have also implemented a scond variant in which the lower-lovel and simplifted $C$ interface is used, as discussed in Sections 3.1 and 3.2 . Finally, a third variant, which we call CC (Callable Continuations), incorporates the proposed improvernents to the model discnssed in Sections 3.3 and 3.4 .

We evaluated the impact of this series of optimizations by asing some of the benchmarks in 'able 1. 'The rosults are shown in l'able 2, whore times are given in milliseconds. Towering the level of the $\mathrm{C}$ interlace and improving the trars formation for tabling and the way calls are performed have a clear impact. It should also be noted that the latter improvement seems to be specially relevant. in non-trivial programs which handle data structures (the larger the data structures are, the more re-copying wo avoid) as opposed to those where little data management is done. On average, we consider the version reported in the rightmost column to be the implomentation of choice among those we have developed, and this is the one we will refer to in the rest of the paper.

'lable 3 tries to determine how the proposed implementation of tabling compares with state-of-the-art systems - namely, the latest available versions of XSB, YapTab, and B-Prolog, at the time of writing. In this table we provide, for several benchmarks, the raw time (in milliscoonds) taken to excoute them using tabling and, when possible, SLD resolution. Measurements have been made 
on Ciao-1.13, using the standard, unoptimized bytecodo-based compilation, and with the CO extensions loaded, as well as in XSB 3.0.1, YapTab 5.1.1, and B-Prolog 7.0. All the executions were performed using local scheduling and disabling garbage colloction; in the end this did not impact excoution times very much. We used gcc 4.1.1 to compile all the systems, and we executed them on a machine with Fedora Core Linux, kernel 2.6.9, and an Intel Xeon processor.

Analyzing the belavior of the rcycle $X$ benchmark, which is an example of almost pure tabling evaluation, we observe that our asymptotic behavior is similar to other tabling approaches. If we multiply $\mathrm{x}$ by $N$, the resulting time for all of the systems (except. YapTab) is multiplied by approximately $2 N$. YapTab doos not follow the same behavior, and, while wo could not find out oxactly the reason, we think it is due to YapTab on-the-/ly creating an indexing table which solects the right edge/ 2 clauso in constant time, while other implomentations spend more time perforrning a search.

B-Prolog, which uses a linear tabling approach, is the fastest SLG resolution implementation for $r c y c l e x$, since there is no rocomputation in that benchmark. However, efliciency suffers if a costly predicate has to be recompaled: this is what happens in rcycleF, where we added a call to a predicato calculating the $20^{\text {th }}$ Fiboracei ntmber before each of the calls to edge/2 in the body of path/2. This is a (well-known) disadvantage of linear tabling techniques which does not affect susponsion-based approachos. It has to bo noted, however, that curent versions of B-Prolog implement an optimized variant of its original lincar tabling mechanism [22] which tries to avoid rocvaluation of looping subgoals. The impact of recomputation is, therefore, not as important, as it may initially seem. Additionally, in our experience B-Prolog is already a very fast SLD system, and its specd secms to carry on to SLC exceution, which makes it, in our experiments, the fastest SIG systern in absolute terms, except when mmeeded recomputation is performed.

The ideas discnssed in Section 3.5 show their eflectiveness in the rcycleR 2048 benchmark, where duplicating the clauses of edge/2 produces repeated consumers. While B-Prolog is affected by a factor close to 2, and XSB and Yap'lab by a factor of 1.5, the Ciao+CC implementation is allected only by a factor of a $5 \%$ because it does not add repeated consumers to the tabled evaluation.

In order to compare onr implementation with XSB, we must take into account that XSB is somewhat slower than Ciao when executing programs using SLD resolution - at least in those cases whore the program oxecution is large enough to be really significant (between 1.8 and 2 times slower for these nontrivial programs). This is partly due to the fact that $\mathrm{XSB}$ is, even in the case of SLD execution, prepared for tabled resolution, and thus the SLG-WAM has an additional overhoad (reported to bo around 10\% [16]) not present in other Prolog systems and also presumably that the priorilies of their implementors were understandably more focused on the implementation of tabling. However, XSB cxcoutes tabling around 1.8 times faster than our current implementation, confiming, as expected, the advantages of the native implementation, since we perform some operations at the l'rolog lovel. 
Table 3. Comparimg Ciao+ OC with XSB, Yap'ab, and B-Prolog

\begin{tabular}{|c|c|c|c|c|c|c|c|c|}
\hline \multirow[b]{2}{*}{ Program } & \multicolumn{2}{|c|}{$\mathrm{Ciac}+\mathrm{CC}$} & \multicolumn{2}{|c|}{ XSB } & \multicolumn{2}{|c|}{ YapTab } & \multicolumn{2}{|c|}{ B-Prolog } \\
\hline & SLD & \begin{tabular}{|l|} 
Tabling \\
\end{tabular} & SLD & \begin{tabular}{|l|} 
Taljling \\
\end{tabular} & SLD & \begin{tabular}{|l} 
Tabling \\
\end{tabular} & SLD & \begin{tabular}{|l} 
Tabling \\
\end{tabular} \\
\hline reycle 256 & - & 70.57 & - & 36.4 | & - & 59.95 & - & 26.02 \\
\hline reycle 512 & - & 288.14 & - & 151.26 & - & 311.47 & - & 103.16 \\
\hline rcycle 1,024 & - & $1,097.26$ & - & 683.18 & - & $1,229.86$ & - & 407.95 \\
\hline reyclo 2,048 & - & 4.375 .93 & - & 3.664 .02 & - & $2,451.67$ & - & $1,596.06$ \\
\hline roycleh 2,048 & - & 4.578 .50 & - & 5.473 .91 & - & 3.570 .31 & - & $2,877.60$ \\
\hline reycleF 256 & - & $1,641.95$ & - & $2,472.61$ & - & $1,023.77$ & - & $2,023.76$ \\
\hline rumbers 3r & 1.62 & 1.39 & 3.61 & 1.91 & 1.87 & 1.08 & 1.16 & 1.13 \\
\hline numbers $4 \mathrm{r}$ & 99.74 & 36.13 & 211.08 & 51.72 & 108.08 & 29.16 & 83.89 & 22.07 \\
\hline numbers 5r & $7,702.03$ & $1,059.93$ & $16,248.01$ & $1,653.82$ & $8,620.33$ & 919.88 & $6,599.75$ & 708.40 \\
\hline aul $r^{2}$ & - & 703.19 & - & 581.31 & - & 278.41 & - & 272.55 \\
\hline
\end{tabular}

Although this lower efficiency is obviously a disadvantage of our implemenlation, it is worth noting that, since our approach does not introduce changes neither in the WAM nor in the associated Prolog compiler, the speed at which non-tabled l'rolog is executed remains unchanged. In addition to this, the modular design of our approach gives better chances of making it easier to port to other systems. In our case, executables which do not need tabling have very little tabling-related code, as the data structures (for tries, elc.) are created as dynamic libraries, loaded on demand, and only stubs are needed in the regular engine. 'The program transformation is taken care of by a package (a plugin for the Ciao compiler) which is loaded and active only at compile time.

In non-trivial benchmarks like numbers $\mathrm{Xr}$, which at least in principle should rellect more accurately what one might expect in larger applicalions, execulion times are in the end somewhat favorable to Ciao $+\mathrm{CC}$ when comparing with XSB. This is probably due to the faster raw speed of the basic engine in Ciao but it also implies that the overhead of the approach to habling used is reasonable after the proposed optimizations. In this context it should be noted that in these experiments we have used the baseline, bytecode-based compilation and abstract. machine. Turning on global analysis and using optimizing compilers [3,10] can further improve the speed of the SLD part of the computation.

The results are also encouraging to us becanse they appear to be another example supporting the "Ciao approach:" start from a fast and robust, but extensible LI'-kemel system and then include additional characteristics by means of pluggable components whose implementation mist, of course, be as efficient as possible but. which in the end benefit. from the initial base speed of the systerm.

We have not analyzed in detail the memory consumption behavior of the continuation call tcchnique, as wo are right now working on improving it. However, since we copy the sarme part of the heap CAT does, but using a different. strategy, and we eventually (as gencrators are completed) get rid of the data structures corresponding to the frozen continuation calls, we foresee that our memory consumption should currently be in the same range as that of CAT. 


\section{Conclusions}

We have reported on the design and efficiency of some improvements made to the continuation call mechanism of Ramesh and Chen. While, as expected, we cannot achieve using just these techniques the same level of performance during tabled evaluation as the natively implemented approaches our experimental results show that the overhead is essentially a reasonable constant factor, with good scaling and convergence characteristics. We argue that this is a useful result since the proposed mechanism is still easicr to add to an existing WAMbased system than implementing other approaches such as the SIG-WAM, as it requires relatively small changes to the underlying execution engine. In fact, almost everything is implemented within a fairly reusable $C$ library and using a I'rolog program transformation. Our main conclusion is that using an cxternal module for implementing labling is a viable alternative for adding tabled evaluation to Prolog systems, especially if coupled with the proposed optimizations. It is also an approach that ties in well with the modular approach to extensions which is an integral part of the design of the Clao systern.

\section{Acknowledgments}

This work was funded in part by the IST program of the European Commission, FP6 FET project IST-15905 MOBWS, by the Spanish Ministry of Fducation and Science (MEC) project TIN2005-09207-C03 MERIT-COMVERS and by the Government of the Madrid Region (CAM) Project S-0505/ I'IC/0407 PROMESAS. Manuel Hermenegildo is also funded in part by the Prince of Asturias Chair in Information Science and Technology at the U. of New Mexico, USA and the IMDE $\Lambda$-Software Institute, Madrid, Spain. Cláudio Silva and Ricardo Rocha were partially funded by project Myddas (POSC/EIA/59154/2004). Ricardo Rocha was also fiunded by project S'I'AMI' $\Lambda$ (I'I'DC/EI $\Lambda / 67738 / 2006)$.

\section{References}

Bucno, F., Gabeza, D., Carro, M., Hemonegildo, M., Tóper-Carcía, P., T'ucbla, G. (eds.): Tho Ciao Systom. Thof. Manual (vI.13). Tochnical report, C. S. School (UPM) (2006), http://ww . ciaohome.org

Cabeza, D., Hermenegildo, M.: The Ciao Modular, Standalone Compiler and Its Gencric Trogram Procossing Tibrary. In: Special Issue on Parallelism and Tmplementation of (C)LP Systems. Llectronic Notes in 'I'heorelical Compuler Science, vol. 30(3), Lilsevier, North Ilolland (2000)

Carro, M., Morales, J., Muller, H.L., Puebla, G., Hemenegildo, M.: High-Level Languages for Small Devices: A Case Study. In: Flautner, K., Kim, T. (eds.) Compilers, $\Lambda$ rchitecture, and Synthesis for Embedded Systoms, pp. 27। 281. ACM Press / Slreridan (October 2006)

Chen, W., Warren, D.S.: Tabled Evaluation with Delaying for General Logic Programs. Jourmal of the $\Lambda$ CM $13(1), 2071$ (1996)

Dawson, S., Ramakrislman, C.R., Warren, D.S.: Practical Progran Analysis Using General Purpose Logic Programming Systems - A Case Study. In: Proceedings of PLDT 1996, pp. II7 I26. ACM I'ress, Now York, USA (1996) 
Chico de Guzmán, P., Carro, M., Ilermenegildo, M., Silva, G., Rocha, R.: Some Improvements over the Continuation Call Tabling Implementation Techique. In: CTCT,OPS 2007, ACM Press, Now York (2007)

Demoen, B., Sagonas, K.: CAT: 'The Copying Appronch to 'labling. In: Palamidessi, C., Meinke, K., Glaser, H. (eds.) ALP 1998 and PLILP 1908. LNCS, vol. 1490, pp. 21-35. Springer, Heidelberg (1998)

Demoen, B., Sagonas, K.F.: Chat: The copy-hybrid approach to tabling. Tractical Applications of Declaralive Languages, 106-121 (1999)

Guo, H.-F., Gupta, G.: A Simple Scheme for Implementing Tabled Logic Programming Systems Based on Dynamic Reordoring of $\Lambda$ tematives. In: Tnternational Conlerence on Logic Programming, pp. 181-196 (2001)

Morales, J., Carro, M., Ilermenegildo, M.: Improving the Compilation of Prolog to O Using Moded I'ypes and Determinism Informalion. In: Jayaraman, L3. (ed.) PADL 2004. LNCS, vol. 3057, pp. 86-103. Springer, Heidelberg (2004)

Ramakrishna, Y.S., Ramakrishnan, C.R., Ramakrishnan, I.V., Smolka, S.A., Swift, 'I', Waren, D.S.: Lilicient Model Checking Using 'Labled Resolution, In: Grunberg, O. (ed.) CAV 1997. LNCS, vol. 1254, pp. 143-154. Springer, Heidelberg (1997) Ramakrishnan, I.V., Rao, P., Sagonas, K.T', Swift, T., Warren, D.S.: Ffficient t.abling mochanisms for logic: programs. Tn: ICLP, pp. 697 71.1 (1995)

Ramakrishnan, R., Ullman, J.D.: A survey of research on deductive database systems. Journal of Logic Programming 23(2), 125-149 (1993)

Ramesh, Tk., Chon, W.: $\Lambda$ Portable: Mothor for Integrating SICG Rcsolution into Prolog Systems. In: Bruynogghe, M. (ed.) International Synposium on Logic Programming, pp. 618-632. MIT Press, Cambridge (1994)

Rocha, R., Silva, O, Isopes, R.: On Applying PTogram Transformation to Implement Suspension-Based Tabling in Prolog. Tn: Dahl, V., Niemdä, J. (cds.) TCLP 2007. LNCS, vol. 4670, pp. 444-445. Springer, lleidelberg (2007)

Sagonas, K., Swift, T.: An Abstract Machine for Tabled Execution of Fixed-Order Stratified Logic Programs. ACM Transactions on Programming Isanglages and Systems 20(3), 586-634 (1998)

Silva, C.: On Applying Program Transformation to Implement Tabled Evaluation in Prolog. Master's thesis, Faculdade de Ciências, Universidade do Porto (January 2007) Somogyi, Z., Sagonas, K.: 'Tabling in Mercury: Design and Innplenentation. In: Vau llemtenryck, P. (ed.) PADL 2006. LNCS, vol. 3819, pp. 150-167. Springer, Heidelberg (2005)

Tamaki, H., Sato, M.: OT.D rosolution with tabulation. In: Third Intornational Conference on Logic Programming, London. LNCS, pp. 84-98. Springer, Ileidelberg (1986) Warren, D.S.: Memoing for logic programs. Communications of the ACM 35(3), $931 \mathrm{II}(1992)$

Waren, R., llermenegildo, M., Debray, S.K.: On the Practicalily of Global flow Analysis of Logic Programs. In: Fifth Internalional Conference and Synposium on Logic Programming, pp. 684-699. MIT Press, Cambridge (1988)

Zhou, N.-F., Sato, T., Shen, Y.-D.: Linear Tabling Strategies and Optimizations. Thoory and Practice of Togic programming (accoted for publication 2007), http: //arxiv.org/abs/0705.3468v1

Zhou, N.-F., Shen, Y.-D., Yuan, L.-Y., You, J.-H.: Implementation of a linear tabling mechanism. Journal of Functional and Logic Programming 2001(10) (Octolser 2001)

Zou, Y., Finin, 'L', Ghen, 11.: F-OWL: An lnference Lingine for Sennalic Web. In: Hinchey, M.G., Rash, J.L., Truszkowski, W.F., Rouff, C.A. (eds.) FAABS 2004. LNCS (INAI), wol. 3228, pp. 238 248. Springer, Heidelberg (2004) 